EPJ manuscript No.

(will be inserted by the editor)

\title{
Low-mass dielectrons from the PHENIX experiment at RHIC
}

\author{
Alexander Kozlov ${ }^{1}$ for the PHENIX collaboration \\ Weizmann Institute of Science, Rehovot 76100, Israel \\ Received: 15.08.2006 / Revised version: 15.08.2006

\begin{abstract}
The production of the low-mass dielectrons is considered to be a powerful tool to study the properties of the hot and dense matter created in the ultra-relativistic heavy-ion collisions. We present the preliminary results on the first measurements of the low-mass dielectron continuum in $A u+A u$ collisions and the $\phi$ meson production measured in $A u+A u$ and $d+A u$ collisions at $\sqrt{s_{N N}}=200 \mathrm{GeV}$ performed by the PHENIX experiment.
\end{abstract}

PACS. 25.75.-q Relativistic heavy-ion collisions - 12.38.Mh Quark-gluon plasma

\section{Introduction}

Among the many diagnostic tools for the hot and dense matter produced in high energy heavy-ion collisions the production of low-mass lepton pair: 1 plays an important role. Dileptons are emitted during the entire lifetime of the collision and interacting only electromagnetically, escape (U) the interaction region almost freely carrying information 1 directly to the detector. This makes dileptons an excel$\checkmark$ lent tool to study the thermal radiation emitted by the dense medium and possible in-medium modifications of - the light vector mesons properties (mass and/or width). This modifications are considered important signals of the restoration of chiral symmetry.

The production of low-mass electron pairs was extensively explored by the CERES collaboration at the SPS 1 . CERES discovered an excess of the dielectron yield in the mass range $0.2-0.6 \mathrm{GeV} / \mathrm{c}^{2}$ as compared to the one expected from the hadronic sources. A similar excess was observed for different colliding energies and species by DLS at the BEVALAC [2], E325 at KEK [3] and recently by the second generation experiments NA60 at the SPS [4] and HADES at GSI [5]. For a summary of the most recent experimental results see [6.

The absolute yield and shape of the low-mass dielectron spectra measured by CERES are described satisfactorily by various theoretical models involving thermal radiation from the $\pi \bar{\pi}$ annihilation and in-medium modifications of the $\rho, \omega$ and $\phi$ vector mesons spectral functions $[7$.

The $\phi$ meson is considered to be a sensitive probe of the possible restoration of chiral symmetry in relativistic heavy-ion collisions which could manifest itself in the modification of its spectral properties (peak position and/or width) and in the changes of the relative yield as measured

\footnotetext{
1 Low-mass dilepton pairs include the light vector mesons and the dielectron continuum with mass $m_{e^{+} e^{-}} \lesssim 1 \mathrm{GeV} / \mathrm{c}^{2}$.
}

through the $e^{+} e^{-}$vs. the $K^{+} K^{-}$decay channels 8,9 . Since $m_{\phi}$ is slightly larger than $2 m_{K}$, even small changes in the spectral properties of the $\phi$ or $K$ can induce significant changes in the branching ratio of the $\phi \rightarrow K^{+} K^{-}$ decay.

The lifetime of the $\phi$ meson is long compared to the lifetime of the fireball at RHIC energies $\left(\tau_{\phi} \approx 46 \mathrm{fm} / \mathrm{c} v \mathrm{~s}\right.$. $\tau_{\text {fireball }} \approx 10 \mathrm{fm} / \mathrm{c}[10]$ ). In this case only a small fraction of the $\phi$ mesons will decay inside the fireball, which could lead to a distortion of the line-shape if the $\phi$ mesons spectral function is affected by the medium. For example, a low-mass tail should develop if the $\phi$-meson mass decreases in the medium.

The PHENIX detector has the potential to measure accurately $e^{+} e^{-}$pairs in the low-mass region and the light vector meson properties. The observation of a $\phi$-meson shape distortion is difficult but could be possible with the excellent mass resolution, of about $1 \%$, of the PHENIX detector in the $e^{+} e^{-}$decay channel provided that a good signal to background, $S / B$, ratio can be achieved. In addition to that, the PHENIX detector has the unique capability of to measure simultaneously the $\phi$-meson production through the $e^{+} e^{-}$and $K^{+} K^{-}$decay channels.

During RHIC run 4 in 2004, PHENIX has collected about $241 \mu \mathrm{b}^{-1}$ integrated luminosity which allowed us to perform a first measurement of the low-mass dielectron continuum and the $\phi$-meson production via $e^{+} e^{-}$and $K^{+} K^{-}$decay channels in $A u+A u$ collisions at $\sqrt{s_{N N}}=$ $200 \mathrm{GeV}$.

In this paper the low-mass dielectron mass spectra measured in $A u+A u$ collisions at $\sqrt{s_{N N}}=200 \mathrm{GeV}$ [11. and the results of the $\phi$-meson production in $d+A u[12$ and $A u+A u$ 13] collisions at $\sqrt{s_{N N}}=200 \mathrm{GeV}$ as determined by the $e^{+} e^{-}$and $K^{+} K^{-}$channels are presented.

The measurement of low-mass $e^{+} e^{-}$pairs with the present PHENIX configuration is challenging due to huge combinatorial background originating from unrecognized 
conversions and $\pi^{0}$ Dalitz decays. Indeed, the $S / B$ ratio measured in $A u+A u$ collisions at $\sqrt{s_{N N}}=200 \mathrm{GeV}$ is of the order of $\sim 1 / 150$ and $\sim 1 / 60$ at $m_{e^{+} e^{-}} \approx 400 \mathrm{MeV} / \mathrm{c}^{2}$ [11] and $\phi$ mass [13, respectively.

A PHENIX upgrade with a Hadron Blind Detector foreseen in 2006 will significantly reduce the combinatorial background, improving the capability of PHENIX to measure low-mass dielectron pairs in heavy-ion collisions. The details of the HBD project are presented below.

\section{The PHENIX experiment}

\subsection{Experimental apparatus}

PHENIX is one of four experiments at the Relativistic Heavy Ion Collider (RHIC) at Brookhaven National Laboratory. Among them, PHENIX is the only one which has the capability to measure low-mass electron pairs. The PHENIX spectrometer [16] consists of two central arms which cover a pseudorapidity range $|\eta|<0.35$ and $2 \times$ $90^{\circ}$ in azimuthal angle. The momentum and charge of the particles are determined using a Drift Chamber (DC) and a Pad Chamber (PC1). Electrons are identified by a Ring Imaging Cherenkov (RICH) detector and by requiring the energy in a Electromagnetic Calorimeter (EMCal) to match the measured momentum of the tracks. Kaons are identified using the timing information from a Time Of Flight (TOF) detector and EMCal which have very good $\pi / \mathrm{K}$ separation in the momentum range $0.3<p<$ $2.5 \mathrm{GeV} / \mathrm{c}$ and $0.3<p<1.0 \mathrm{GeV} / \mathrm{c}$, respectively. Valid DC-PC1 tracks are confirmed by the matching of the associated hit information to the RICH and EMCal in the case of electrons and to the TOF or EMCal in the case of kaons.

The beam-beam counters $(\mathrm{BBC})$ are used to determine the $z$-coordinate of the collision vertex $\left(z_{v t x}\right)$ and in combination with the zero-degree calorimeters (ZDC) provide the trigger and determine the event centrality.

In order to benefit from the high luminosity of RHIC and provide efficient detection of the rare electron and dielectron events PHENIX successfully implemented an electron trigger (ERT) which requires spatial matching between EMCal and RICH and an energy above a certain threshold in the EMCal.

\subsection{Electron pair analysis with PHENIX spectrometer}

The electron pair analysis is performed with the sample of identified electrons using a statistical procedure in which all particles in a given event are combined into pairs to generate unlike- and like-sign invariant mass spectra. By construction the unlike-sign spectrum contains both the signal and an inherent combinatorial background of uncorrelated pairs. The size and shape of the combinatorial background are determined using the event mixing technique in which the particles from one event are combined with the particles from different events provided that all events belong to the same centrality and vertex classes.
The unlike-sign mixed event integral yield is normalized to the measured $2 \times \sqrt{N^{++} N^{--}}$yield. All yields are calculated above $m_{e^{+} e^{-}}=200 \mathrm{MeV} / \mathrm{c}^{2}$ to exclude the correlated $e^{+} e^{+}$and $e^{-} e^{-}$pairs from double $\pi^{0}$ Dalitz decays and double conversions. PHENIX has refined the mixed-event technique to a very high precision of less than $\pm 0.1 \%$ which is confirmed by comparing the mixed event like-sign invariant mass spectra to the measured one. The normalization procedure has been tested in four different approaches and found to be stable within $0.5 \%$ 11. Finally, the signal mass distribution is derived by subtracting the normalized mixed event spectrum from the measured one.

\section{$3 \phi$-meson production}

The $\phi$-meson yield $(d N / d y)$ and temperature $(T)$ were derived from the invariant $m_{T}$-distribution:

$$
\frac{1}{2 \pi m_{T}} \frac{d^{2} N}{d m_{T} d y}=\frac{N_{r a w}^{\phi}\left(m_{T}\right) \cdot C F\left(m_{T}\right) \cdot \epsilon_{\text {trigger }}\left(m_{T}\right)}{2 \pi m_{T} \cdot N_{\text {events }} \cdot \epsilon_{e m b} \cdot \epsilon_{r b r} \cdot B R \cdot \Delta m_{T}}
$$

where $N_{\text {raw }}^{\phi}\left(m_{T}\right)$ is the raw $\phi$ yield, $C F\left(m_{T}\right)$ is the correction factor to account for acceptance and pair reconstruction efficiency, $\epsilon_{\text {trigger }}\left(m_{T}\right)$ is the electron trigger efficiency which is equal to one for the analysis of minimum bias events, $N_{\text {events }}$ is the number of analyzed events, $\epsilon_{e m b}$ is the pair embedding efficiency which accounts for the reconstruction efficiency losses due to detector occupancy, $\epsilon_{r b r}$ is an efficiency due to run-by-run variations of the detector performance. $B R$ is the branching ratio for $\phi \rightarrow e^{+} e^{-}$or $K^{+} K^{-}$and $\Delta m_{T}$ is the bin size.

The raw $\phi$ yield, $N_{\text {raw }}^{\phi}$, is derived by summing the content of the spectrum over a mass interval of $\pm 3 \sigma_{\text {tot }}$ where $\sigma_{\text {tot }}$ is the total width calculated from the quadrature sum of the experimental mass resolution and natural width of the $\phi$ meson.

The correction factor $C F\left(m_{T}\right)$ is determined using a Monte Carlo simulation. Single $\phi$ mesons were generated with an exponential transverse momentum distribution. The $\phi$ 's are decayed, propagated through an emulator of the PHENIX detector and the resulting output is passed through the whole analysis chain. For each $m_{T}$ bin the ratio of the generated yield to the reconstructed one gives the correction factor $C F\left(m_{T}\right)$.

Finally, the $d N / d y$ and $T$ are extracted from the corrected invariant $m_{T}$ spectra fitted with the following exponential function, having $d N / d y$ and $T$ as parameters:

$$
\frac{1}{2 \pi m_{T}} \frac{d^{2} N}{d m_{T} d y}=\frac{d N / d y}{2 \pi T\left(T+M_{\phi}\right)} \exp \frac{-\left(m_{T}-M_{\phi}\right)}{T},
$$

where $M_{\phi}$ is the PDG value of the $\phi$-meson mass.

\section{$3.1 d+A u$ collisions}

The measurements in $d+A u$ collisions establish the baseline information for possible nuclear matter effects and 
Table 1. $d N / d y$ and $T$ for the $\phi \rightarrow e^{+} e^{-}$and $\phi \rightarrow K^{+} K^{-}$analysis in $d+A u$ collisions.

\begin{tabular}{lll}
\hline Decay channel & $d N / d y$ & $T(M e V)$ \\
\hline$\phi \rightarrow e^{+} e^{-}$ & $0.056 \pm 0.015$ (stat) \pm 0.028 (syst) & $326 \pm 94$ (stat) \pm 118 (syst) \\
$\phi \rightarrow K^{+} K^{-}$ & $0.047 \pm 0.009$ (stat) \pm 0.010 (syst) & $414 \pm 13$ (stat) \pm 23 (syst) \\
\hline
\end{tabular}

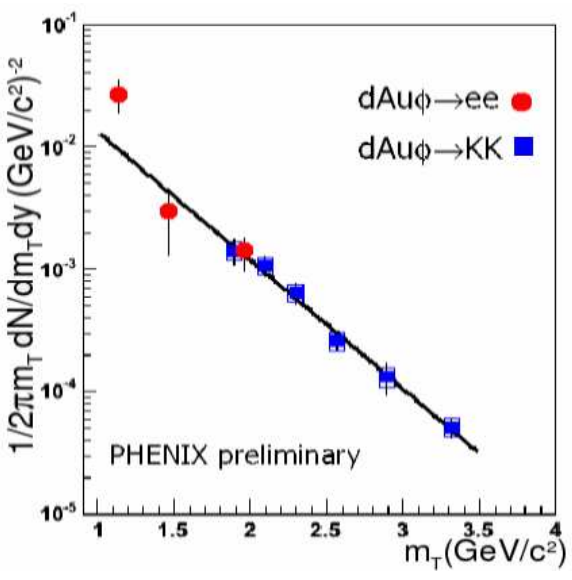

Fig. 1. (Color online) $m_{T}$ spectra of the $\phi$ mesons from the $\phi \rightarrow e^{+} e^{-}$and $\phi \rightarrow K^{+} K^{-}$analyses in $d+A u$ collisions. Statistical and systematic errors are shown by vertical bars and open rectangles, respectively. The line represents the common fit to the exponential function, Eq. 2 .

provide an essential reference for the comparison to the measurements in $A u+A u$ collisions.

The presented results are based on about 31 million triggered $d+A u$ events with a threshold of $600 \mathrm{MeV}$ (see Sec. 2.1) and about 62 million minimum-bias $d+$ $A u$ events for the $\phi \rightarrow e^{+} e^{-}$and $\phi \rightarrow K^{+} K^{-}$analyses, respectively.

The $\phi$-meson invariant $m_{T}$ spectra from both $e^{+} e^{-}$ and $K^{+} K^{-}$analyses are shown in Fig. 1 by the filled circles and squares, respectively 12 . The line represents the fit to all the data points with the exponential function given by Eq. 2 .

$d N / d y$ and temperatures extracted from the $\phi \rightarrow e^{+} e^{-}$ and $\phi \rightarrow K^{+} K^{-}$analyses in $d+A u$ collisions are listed in Table 1 .

\section{$3.2 A u+A u$ collisions}

In this section we present the $m_{T}$ spectra, invariant yield and temperature results for the different centralities derived from the measurements of the $\phi$ meson through the $e^{+} e^{-}$and $K^{+} K^{-}$decay channels in $A u+A u$ collisions at $\sqrt{s_{N N}}=200 \mathrm{GeV}$. The $\phi \rightarrow e^{+} e^{-}$analysis uses $903 \mathrm{mil}-$ lion minimum-bias events. Kaons are combined in pairs using different combinations of the TOF and EMCal used for the kaon identification (see Sec. 2.1). The analysis performed with $409 \times 10^{6}$ minimum-bias events for TOF-TOF and $170 \times 10^{6}$ minimum-bias events for TOF-EMCal and EMCal-EMCal detector combinations.
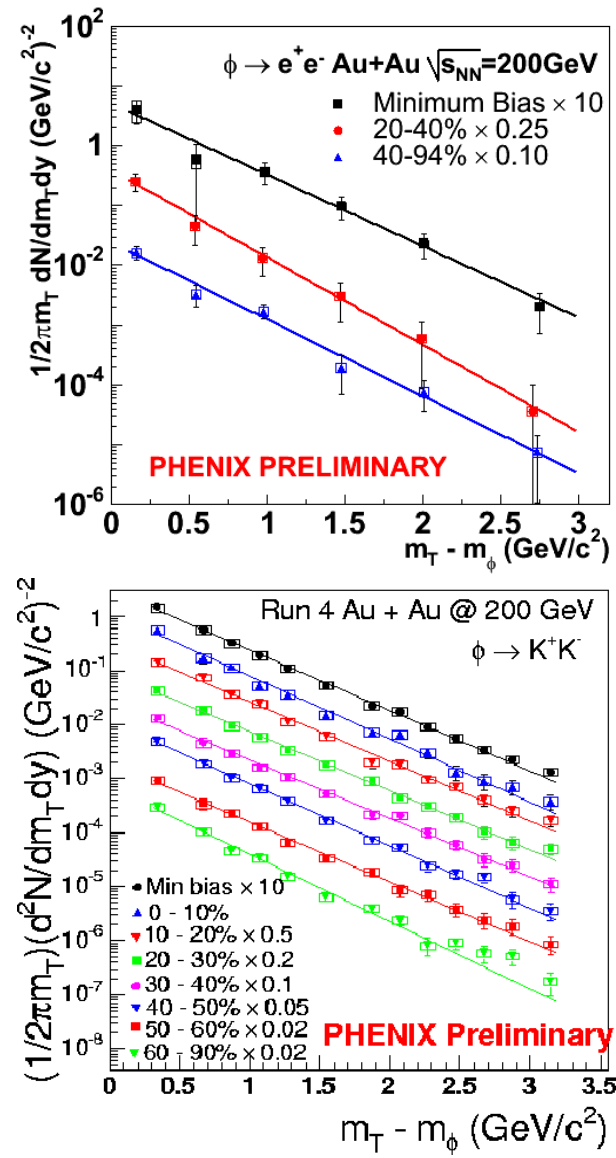

Fig. 2. (Color online) Invariant $m_{T}$ spectra of the $\phi \rightarrow e^{+} e^{-}$ (top) and $\phi \rightarrow K^{+} K^{-}$(bottom) for minimum bias and several centrality bins in $A u+A u$ collisions. Statistical and systematic errors are shown by vertical bars and open rectangles, respectively. Each line represents the fit to the exponential function, Eq. 2,

The invariant $m_{T}$ spectra for minimum bias and several centrality bins, fitted with the exponential function Eq. 2. are shown in the top and bottom panels of Fig. 2 for $\phi \rightarrow e^{+} e^{-}$and $\phi \rightarrow K^{+} K^{-}$analyses, respectively.

Fig. 3 shows the centrality dependence of the $\phi$-meson yield per participant pair extracted from the $m_{T}$ distributions in both analyses. The highest centrality bin shown by the triangle in the left panel of Fig. 3 has limited statistics and is derived from an independent analysis. In this analysis the invariant yield of the $\phi$ meson is obtained using the integral yield and the correction factor integrated over $m_{T}$ assuming $T=366 \mathrm{MeV}$.

The yield per participant pair shows a $\sim 50 \%$ increase in the $K^{+} K^{-}$decay channel from peripheral to central 

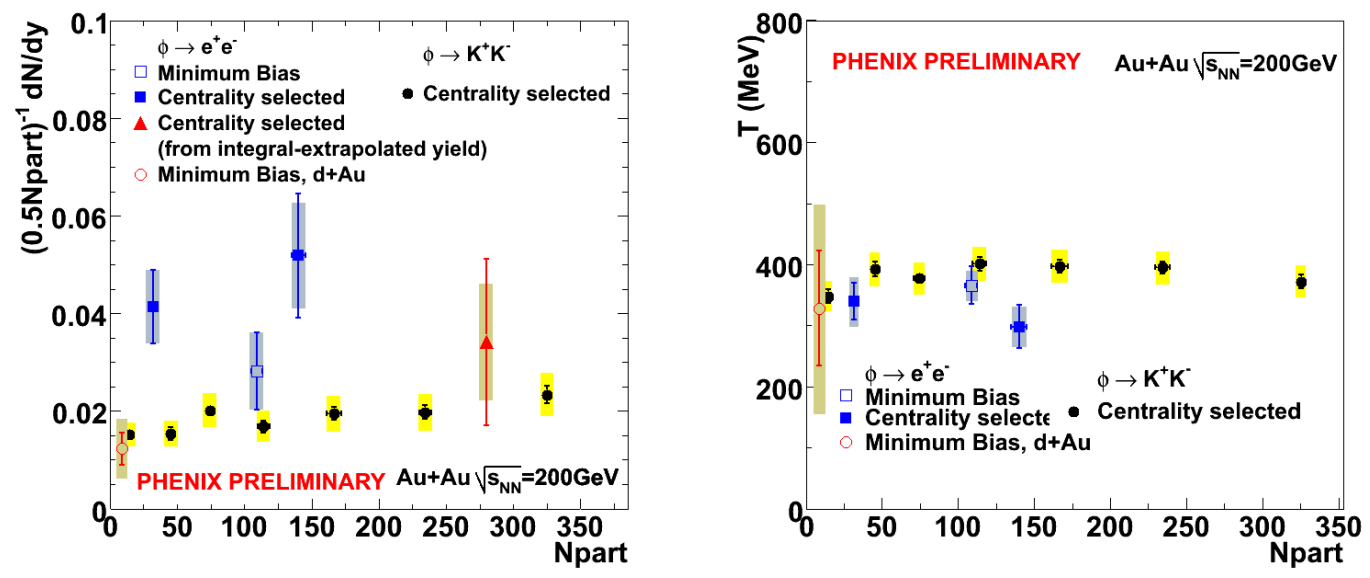

Fig. 3. (Color online) Multiplicity dependence of the $\phi$-meson yield normalized to number of participant pairs, $\left(0.5 \cdot N_{\text {part }}\right)^{-1}$ $d N / d y e^{+} e^{-}$and $K^{+} K^{-}$decay channels. Open and filled squares represent minimum bias and centrality selected events, respectively. The triangle shows the $\phi$ yield derived from an independent analysis. The open circle represents the reference measurements of $d N / d y$ in $d+A u$ collisions. Statistical and systematic errors are shown by vertical bars and shaded bands, respectively.

collisions. The present statistical and systematic uncertainties do not allow us to infer the centrality dependence of the yield measured in the $e^{+} e^{-}$decay channel (left panel of Fig. 3]).

The comparison of the $\phi$-meson production measured via the $e^{+} e^{-}$and $K^{+} K^{-}$decay channels shown in Fig. 3 may indicate a possible increase of the yield in the dielectron channel compared to the kaon one. However, the statistical and systematic errors in the dielectron channel are too large for a definite statement and within the error bars the yield in the two decay channels are consistent. The temperatures measured in $A u+A u$ collisions through the $e^{+} e^{-}$and $K^{+} K^{-}$decay channels (see right panel of Fig. 3) are centrality independent and agree within the statistical and systematic uncertainties.

The open circles in Figs. 3. represent the reference measurements of the yield and $T$ in $d+A u$ collisions and both are found to be in a good agreement with $d N / d y$ and $T$ measured in the peripheral $A u+A u$ collisions via the $K^{+} K^{-}$decay channel. The temperatures measured via the $e^{+} e^{-}$decay channel in $d+A u$ and $A u+A u$ collisions are consistent within the error bars. The yield per participant pairs measured in $d+A u$ collisions is about two times smaller than the one measured in peripheral $A u+A u$ collisions in the $e^{+} e^{-}$channel but the large statistical and systematic uncertainties do not allow for a conclusive statement.

\section{Low-mass dielectron continuum}

The results presented in this section are based on the analysis of 800 million minimum-bias events collected in $A u+A u$ collisions at $\sqrt{s_{N N}}=200 \mathrm{GeV}$.

Fig. 4 shows the foreground, background, and subtracted invariant mass spectra of the dielectron pairs. The

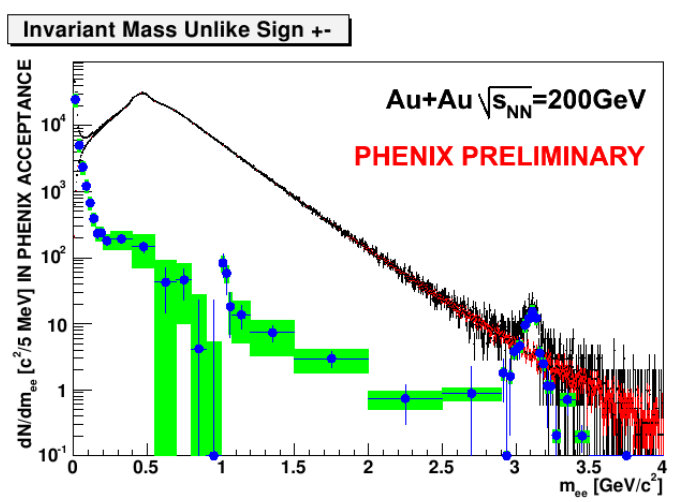

Fig. 4. (Color online) The foreground (black), background (red) and subtracted $e^{+} e^{-}$invariant mass spectra. Statistical and systematic errors are shown by vertical bars and shaded bands, respectively.

background distribution was obtained with an event mixing procedure with remarkable precision (see Sec. 2.2). One can see that the signal to background ratio, $S / B$, at masses $m_{e^{+} e^{-}} \approx 400 \mathrm{MeV} / \mathrm{c}^{2}$ is of the order of $\sim 1 / 150$. With such a small $S / B$ ratio the systematic errors, shown in Fig. 4 by shaded bands, are dominated by the normalization of the background spectrum.

Fig. 5] shows the comparison of the measured $e^{+} e^{-}$invariant mass spectrum to the expected cocktail of hadron decays and to the theoretical predictions from the $\pi \bar{\pi}$ annihilation channel without (dashed line) and with (dotted and dashed-dotted lines) modification of the $\rho$-meson spectral function 14,15. The results shown in Fig. 5 may indicate an enhancement of the dielectron yield in the mass range between 0.2 to $0.6 \mathrm{GeV} / \mathrm{c}^{2}$ over the expected hadronic cocktail and even the calculations involving in- 


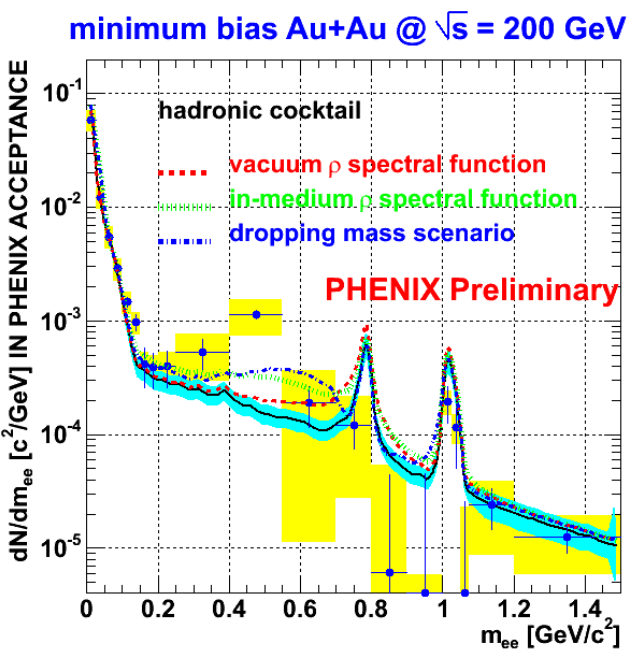

Fig. 5. (Color online) The measured $e^{+} e^{-}$invariant mass spectrum compared to the hadronic cocktail (solid line) and calculations assuming the vacuum $\rho$ spectral function (dashed), in-medium broadening of the $\rho$ width (dotted) or $\rho$ dropping mass (dashed-dotted).

medium modification of the $\rho$ meson, although the present uncertainties do not allow us to draw a strong conclusion.

Fig. 6] shows the measured $e^{+} e^{-}$spectra for the central $(0-20 \%)$ and semi-peripheral $(20-92 \%)$ centrality classes compared to the hadronic cocktail. The possible excess of $e^{+} e^{-}$pairs within mass region $0.2-0.6 \mathrm{GeV} / \mathrm{c}^{2}$ is seen in the central collisions whereas no excess is seen in the semi-peripheral ones.

The in-medium modification of the dielectron continuum can be studied also by looking at the ratio of the $e^{+} e^{-}$yield in different mass regions with respect to the $\pi^{0}$ Dalitz region $\left(m_{e^{+} e^{-}}<100 \mathrm{MeV} / \mathrm{c}^{2}\right)$ which is independent of the number of participants. The left panel of Fig. 7 shows the ratio of the yield in the mass region between 150-450 $\mathrm{MeV} / \mathrm{c}^{2}$ overlaid with the pion yield per participant pair shown by the crosses [17. The pion yield scales with $N_{\text {part }}$ while the ratio could have an indication of nonlinearity. The ratio for the mass region 1.2-2.9 $\mathrm{GeV} / \mathrm{c}^{2}$ (right panel) is within the uncertainties independent of $N_{\text {part }}$ although it is expected to increase following the scaling with the number of participants of the charm production [18] which is the main source of the dielectrons in this mass region. The statistical significance of these results, however, is limited due to the low $S / B$ ratio and does not allow us to draw a conclusion.

\section{The Hadron Blind Detector}

The capability of the PHENIX detector to measure lowmass dielectron pairs will be greatly improved with an upgrade that will add a Hadron Blind Detector (HBD) [19].

The detector is a conceptually new Čerenkov detector operated with pure $\mathrm{CF}_{4}$ in a proximity focus configuration. It is coupled directly to a triple Gas Electron Multiplier $(G E M)$ 21] detector with a CsI photocathode layer
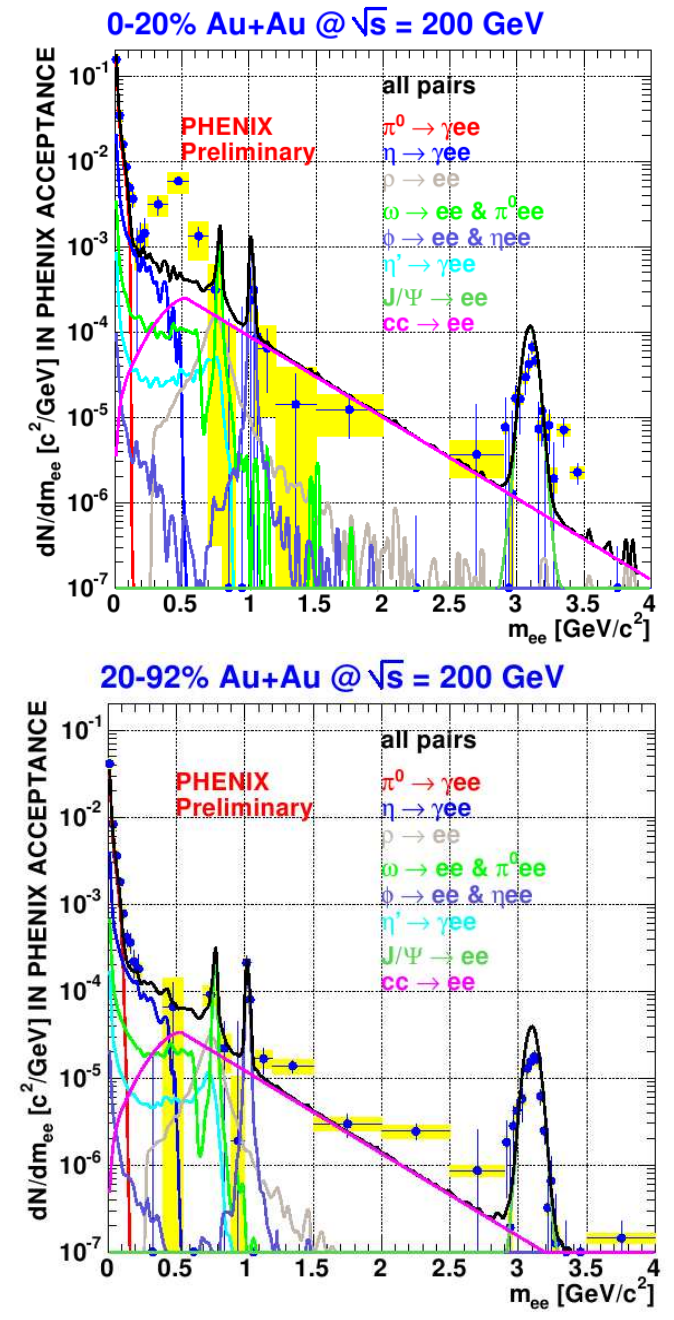

Fig. 6. (Color online) The comparison of the data to a hadronic cocktail and charm contribution for centrality classes 0-20\% (right) and 20-92\% (left). Statistical and systematic errors are shown by vertical bars and shaded bands, respectively.

evaporated on the top face of the first GEM foil. The detector has a pad readout scheme.

The HBD is located in the field free region extending up to $r \leq 60 \mathrm{~cm}$ of the inner part of the PHENIX detector (Fig. 8), which is realized by running the inner coil, recently installed in PHENIX for this purpose, with opposite current to compensate for the field from the outer coil. Čerenkov photons from an electron passing through the radiator are directly collected on the CsI photocathode. In this configuration photons form a circular blob, not a ring as in a RICH detector. The photoelectrons emitted from the photocathode are amplified by the triple $G E M$ detector. Finally, the electron avalanche is read out in a pad plane located at the bottom of the detector element.

The HBD exploits the fact that the opening angle of electron pairs from $\gamma$ conversions and $\pi^{0}$ Dalitz decays is small compared to that of pairs from the light vector mesons. In the field-free region this angle is preserved and by applying an opening angle cut one can reject more than 

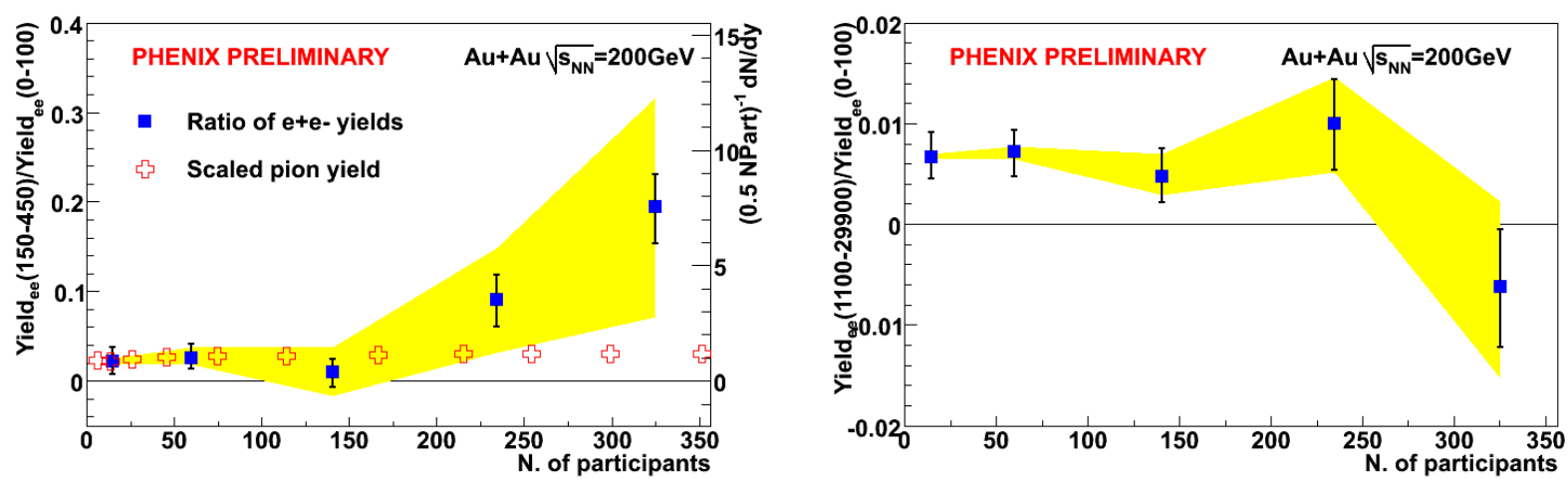

Fig. 7. Ratio of the dielectron yield measured in the mass region 150-450 $\mathrm{MeV} / \mathrm{c}^{2}$ (left) and 1.1-2.9 $\mathrm{GeV} / \mathrm{c}^{2}$ (right) with respect to the $\pi^{0}$ yield $\left(m_{e^{+} e^{-}}<100 \mathrm{MeV} / \mathrm{c}^{2}\right)$. Crosses represent the pion yield per participant pair as a function of the number of participants [17. Statistical and systematic errors are shown by vertical bars and shaded bands, respectively.

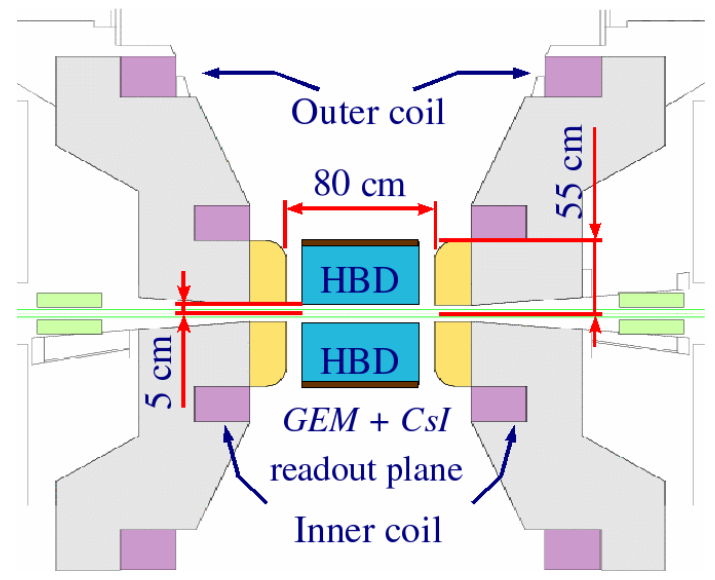

Fig. 8. Layout of the inner part of the PHENIX detector.

$90 \%$ of the conversions and $\pi^{0}$ Dalitz decays, while keeping most of the signal.

The rejection ability of the HBD is confirmed by realistic Monte-Carlo simulations which demonstrated that with the HBD the combinatorial background originating from conversions and $\pi^{0}$ Dalitz decays is reduced by approximately two orders of magnitude.

A comprehensive $R \& D$ program demonstrated the feasibility of the proposed concept 20]. The HBD construction and installation are nearing completion with first operation foreseen at the next RHIC run 22 .

\section{Acknowledgments}

The author acknowledges support by the Israel Science Foundation, the MINERVA Foundation and the Nella and Leon Benoziyo Center of High Energy Physics Research.
2. R. J. Porter et al., Phys. Rev. Lett. 79, (1997) 1229.

3. K. Ozawa et al., Phys. Rev. Lett. 86, (2001) 5019.

4. R. Arnaldi et al., Phys. Rev. Lett. 96, (2006) 162302.

5. G. Agakichev et al., nucl-ex/0608031.

6. I. Tserruya, Nucl. Phys. A774, (2006) 415.

7. R. Rapp, J. Wambach, Adv. Nucl. Phys. 25, (2000) 1, H. van Hees, R. Rapp, hep-ph/0603084.

8. D. Lissauer, E. V. Shuryak, Phys. Lett. B253, (1991) 15.

9. S. Pal, C.M. Ko and Z. Lin, Nucl. Phys. A707, (2002) 525.

10. J. W. Harris and B. Muller, Ann. Rev. Nucl. Part. Sci. 46, (1996) 71.

11. A. Toia et al. (PHENIX Collaboration) Proceedings of Hot Quarks 2006, to be published in Eur. Phys. J. C.

12. R. Seto et al. (PHENIX Collaboration) J. Phys. G30, (2004) S1017.

13. A. Kozlov et al. (PHENIX Collaboration) Nucl. Phys. A774, (2006) 739.

14. R. Rapp, Proceedings of Winter Workshop on Nuclear Dynamics, 2002, to be published in Heavy Ion Physics, nucl-th/0204003.

15. R. Rapp, Phys. Rev. C63, (2001) 054907.

16. K. Adcox et al. (PHENIX Collaboration), Nucl. Instr. and Meth. A499, (2003) 469.

17. S. S. Adler et al. (PHENIX Collaboration), Phys. Rev. C69, (2004) 034909.

18. S. S. Adler et al. (PHENIX Collaboration), Phys. Rev. Lett. 94, (2005) 082301.

19. Z. Fraenkel, B. Khachaturov, A. Kozlov, A. Milov, D. Mukhopadhyay, D. Pal, I. Ravinovich, I. Tserruya and S. Zhou, The PHENIX Collaboration Technical Note 391, http://www.phenix.bnl.gov/phenix/WWW/publish/tserruya/1-06-HBD-pr 20. A. Kozlov, et al. Nucl. Instr. and Meth. A523, (2004) 345,

Z. Fraenkel et al. Nucl. Instr. and Meth. A546, (2005) 466,

I. Ravinovich et al. Nucl. Phys. A774, (2006) 903.

21. F. Sauli, Nucl. Instr. and Meth. A386, (1997) 531.

22. I. Tserruya, Nucl. Instr.and Meth. A563, (2006) 333.

\section{References}

1. G. Agakichev et al., Eur. Phys. J. C41, (2005) 475,

D. Adamova et al., Phys. Rev. Lett. 91, (2003) 042301. 